\title{
Essais
}

ESSAIS

Revue interdisciplinaire d'Humanités

$16 \mid 2020$

Politicité de la littérature et des arts contemporains

\section{« Dans l'esprit des hommes ». La religion comme expérience collective chez Machiavel}

Sandro Landi

\section{OpenEdition}

1 Journals

Édition électronique

URL : http://journals.openedition.org/essais/1261

DOI : 10.4000/essais. 1261

ISSN : 2276-0970

Éditeur

École doctorale Montaigne Humanités

Édition imprimée

Date de publication : 15 septembre 2020

Pagination : 135-150

ISBN : 979-10-97024-08-6

ISSN : 2417-4211

Référence électronique

Sandro Landi, « «Dans l'esprit des hommes ». La religion comme expérience collective chez

Machiavel », Essais [En ligne], 16 | 2020, mis en ligne le 28 septembre 2020, consulté le 12 octobre

2020. URL : http://journals.openedition.org/essais/1261 ; DOl : https://doi.org/10.4000/essais.1261 


\section{«Dans l'esprit des hommes ». La religion comme expérience collective chez Machiavel}

Sandro Landi

Il y a environ cinquante ans, un grand spécialiste d'histoire religieuse, Delio Cantimori, affirmait que dans les écrits de Machiavel, "le thème religieux " est celui qui est "le moins explicitement défini et le moins clairement expliqué ${ }^{1}$ ». Malgré cela, la question de la religion chez Machiavel est devenue progressivement un thème classique d'étude et de débat ${ }^{2}$. Dans cet article, je me propose de l'aborder sous un angle qui n'a pas été suffisamment exploré même s'il constitue l'un des plus constants et récurrents objets d'analyse et d'écriture de Machiavel : celui de la religion comme expérience ou, plus précisément, comme noyau d'expériences partagées, dans un temps et dans un espace historique déterminés, par une communauté ou par un collectif. À ce sujet, deux précisions s'imposent : en premier lieu, l'expérience religieuse, décrite par Machiavel comme expérience collective, inclut des phénomènes qui transcendent le thème religieux au sens strict et donc les quelques écrits - comme les chapitres 11-15 du premier livre des Discours - qui lui sont explicitement consacrées. En second lieu, le moment est sans doute venu d'affirmer que la question, controversée et insoluble, de la religiosité/irréligiosité de Machiavel n'est pas pertinente pour une interprétation correcte de son jugement sur le phénomène religieux.

1 Delio Cantimori, "Niccolò Machiavelli: il politico e lo storico ", in Storia della Letteratura italiana, IV, Il Cinquecento, Milan, Garzanti, 1966, p. 50.

2 La bibliographie est vaste, outre à celle citée dans ces notes, je renvoie à Emanuele CutinelliRèndina, "Religione ", in Enciclopedia machiavelliana, sous la direction de Gennaro Sasso et Giorgio Inglese, vol. 2, Rome, Istituto dell'Enciclopedia italiana, 2015. p. 392-400 et Alison Brown, "Philosophy and religion in Machiavelli ", in John M. Najemy (éd.), The Cambridge Companion to Machiavelli, Cambridge University Press, 2010, p. 157-172. 


\section{Terrifier}

"Terrifier " (sbigottire) $)^{3}$ est sans doute l'un des termes qui définit le mieux la densité de cette expérience. Nous le retrouvons, à des années de distance, utilisé par Machiavel pour décrire l'état d'âme de ceux qui assistent à un sermon. Dans la lettre à Ricciardo Becchi (1498), Savonarole tente d'agréger ses propres partisans et de déstabiliser ses opposants en agitant l'image de la tyrannie (" en maudissant ses adversaires et en les terrifiant [sbigottirgli] au nom d'un tyran $\left.{ }^{4} »\right)$. Ensuite dans le récit d'un sermon apocalyptique prononcé à Santa Croce par un frère ermite, Francesco da Montepulciano (1513) : Machiavel est ici témoin indirect de l'événement (" je n’ai pas entendu le sermon, car je ne suis pas familier avec de telles pratiques, mais je l'ai entendu réciter de cette façon dans toute Florence ") mais témoin direct de ses effets (" ces choses m'ont à tel point terrifié [sbigottito], que je devais aller ce matin avec la Riccia, et je n'y suis pas allé5 "). Dans un contexte similaire, l'effroi, comme sentiment partagé, est mentionné dans le Chant des Ermites (datable de 1524) : "Sur les hauts sommets de notre Apennin / nous sommes moines et ermites ; / nous voici venu dans cette cité, / parce que astrologues et devins / vous ont tous terrifiés (sbigottiti) / selon que nombre de gens nous ont $\mathrm{dit}^{6}$ ". Frayeur et stupéfaction que Machiavel analyse dans sa double dimension de but recherché par ceux qui prêchent, mais aussi comme condition réellement vécue par un public de fidèles. L'effroi, aussi bien comme procédé que comme expérience, est directement en relation avec le sacré mais Machiavel l'étudie à partir de différentes formes de regroupement. La plupart des cas d'effroi sont en effet liés à des épisodes de la vie militaire.

Dans l'Art de la guerre et dans les Discours, les chefs militaires se montrent capables de déstabiliser l'ennemi en faisant " naître quelque chose qui le terrifie ", comme des rumeurs ou de fausses images de renforts, " de telle sorte que l'ennemi, trompé par cette apparence soit terrifié et, terrifié, puisse être aisément vaincu ${ }^{7}$ ». Â l'inverse, il est indispensable que les commandants sachent dissimuler les causes fortuites de désarroi et neutraliser les « inventions » mises

3 Sbigottire signifie littéralement provoquer du sbigottimento c'est-à-dire un état de désarroi profond et d'angoisse ; je traduis donc sbigottire par terrifier et sbigottimento par effroi.

4 Nicolas Machiavel, Euvres, édition établie par Christian Bec, Paris, Laffont, 1996, p. 1229.

5 Niccolò Machiavelli, Lettere a Francesco Vettorie a Francesco Guicciardini, édition de Giorgio Inglese, Milan, Rizzoli, 1989, p. 204 ; lettre du 19 décembre 1513 ; sur la prédication apocalyptique de Francesco da Montepulciano, Michele Lodone, "The Apocalypse in Florence. Friar Francesco da Montepulciano's Sermon (S. Croce, $18^{\text {th }}$ December 1513) ", in Das bedrohte Eigene - das bedrohlich Fremde, Villa Vigoni, 2016, 14-17 novembre, à paraître, cf. Ottavia Niccoli, Profeti e popolo nell'Italia del Rinascimento, Rome-Bari, Laterza, 1987, p. 133-139.

6 Machiavel, Euvres, p. 1079 ; sur ce texte, cf. Delio Cantimori, « Niccolò Machiavelli », cit., p. 32.

7 Ibid., p. 548 (Art de la guerre, IV). 
en place à cet effet par l'ennemi ${ }^{8}$. Dans les deux cas, s'applique la constatation du caractère facilement altérable d'un groupe soumis à une menace extérieure réelle ou imaginaire?. Dans des cas d'extrême gravité, où la survie même du groupe est en cause, le sacrifice de ses propres hommes (" en livrer une partie en pâture à l'ennemi ») fonctionne comme un traumatisme calculé qui, provoquant des sentiments opposés (" soit les refroidir, soit les réchauffer »), permet de corroborer la foi des survivants (" afin que ceux qui ont ou n'ont pas combattu, vous croient $\left.{ }^{10} »\right)$. De même, dans l'un des chapitres des Discours consacrés à la religion romaine archaïque (I, 15), Machiavel accorde une attention particulière au rituel sophistiqué ("spectacle ») mis en œuvre, selon Tite-Live, par les Samnites de manière à bouleverser les soldats, " avec des paroles de malédiction et en des termes épouvantables ${ }^{11}$ " et à réactualiser brutalement, par le serment collectif, le pacte qui lie la multitude à ses dirigeants et aux dieux.

Dans tous ces cas, l'effroi se prête à une double lecture : d'une part, il est le résultat d'un artifice, mis en œuvre pour créer la panique par des cris, des nouvelles, des exécutions, des défaites réelles ou simulées; d'autre part, c'est une expérience : perception soudaine et traumatique du sacré, qui renouvelle dans la mémoire d'un groupe la peur originelle sur laquelle repose le phénomène religieux, lequel n'est, au fond, que "crainte de Dieu ", comme on peut le lire notamment dans Discours I, $11^{12}$. Dans sa nature de remède extrême (" quand les persuasions ne suffisent pas ${ }^{13}$ »), l'effroi met donc en lumière et réactive une attitude à la croyance qui semble appartenir, de façon authentique, selon Machiavel, à toute forme de groupement humain ${ }^{14}$.

La forme ordinaire de gouvernement de cette disposition naturelle repose sur la persuasion, la technique - ou plutôt l'arsenal de techniques - dont la maîtrise est considérée par Machiavel, déjà dans une lettre d'octobre 1499, comme un signe certain de " prudence $»^{15}$. Le principe qui justifie la persua-

8 Ibid., p. 409 (Discours, III, 14).

9 Ibid., p. 408 : « la multitude est inutile à la guerre, ne fût-ce que parce que cheque rumeur, chaque mot, chaque bruit l'inquiètent et la mettent en fuite ».

10 Ibid., p. 555 (Art de la guerre, IV).

11 Ibid., p. 222 ; pour une lecture de ce rite à la lumière des considérations de Georges Dumezil, La religion romaine archaïque, Paris, Payot, 1974, voir William E. Klein, " Machiavelli, Thucydides, and the Anglo-American tradition ", in Patricia Vilches et Gerald Seaman (éds), Seeking Real Truths: Multidisciplinary Perspectives on Machiavelli, Leiden-Boston, Brill, 2007, p. 389-420.

12 Machiavel, cit., p. 213.

13 Ibid., p. 555 (Art de la guerre, IV).

14 Pour une mise en perspective de la notion de croyance en sciences humaines, mais qui ne prend pas en compte les analyses des acteurs du passé, cf. Christophe Grellard, "Les ambiguïtés de la croyance ", Socio-anthropologie [En ligne], 36 | 2017, mis en ligne le 27 novembre 2017, consulté le 31 janvier 2020 : http://journals.openedition.org/socioanthropologie/ 3121.

15 Lettre d'octobre 1499 à un chancelier de la République de Lucques, in Niccolò Machiavelli, 
sion est d'abord l'existence d'un écart cognitif qui sépare le petit nombre du grand nombre ; on lit ainsi dans Discours I, 11, que " nombreux sont les principes connus d'un sage qui ne portent pas en eux de raisons assez évidentes pour persuader les autres ${ }^{16}$ ". Des raisons inaccessibles à la majorité, donc, patrimoine prudentiel d'une minorité qui est définie dans Discours I, 12, tantôt comme "chefs d'une république ou d'un royaume ", tantôt comme " connaisseurs de choses naturelles », ou " hommes sages ». La connaissance du bien commun, qui semble échapper à la raison ordinaire, rend en substance légitime l'utilisation, par cette minorité, de techniques déceptives dans un but de persuasion collective.

\section{Persuader}

La persuasion est un processus analysé en divers lieux par Machiavel jamais isolément, mais toujours en relation, explicite ou implicite, avec une donnée complémentaire : la " crédulité " d'un groupe. En substance, à la volonté de convaincre, fondée sur des raisons supérieures et cachées, correspond, de façon symétrique, la volonté de croire, fondée, au contraire, sur des "raisons évidentes ". Il est donc nécessaire de souligner que, dans cette perspective, la persuasion n'est jamais pour Machiavel une opération unidirectionnelle et que son résultat, toujours incertain, répond à des exigences de crédibilité, c'est-àdire qu'il doit être conforme à ce que le peuple considère comme digne d'être cru. Dans le Prince (chapitre 6), le caractère éphémère de la persuasion est mis en relation à la " nature changeante " du peuple, tant avide de nouveauté que réticent à trouver des objets stables de croyance ${ }^{17}$. Dans Discours I, 11, au sujet de Savonarole, l'acte de la persuasion est en lien direct avec l'historicité des attentes et des représentations propres à chaque peuple (" quoiqu'il soit plus facile de persuader des hommes rudes des vertus d'une institution ou d'une opinion nouvelle, il n'est pas pour autant impossible d'en persuader les hommes civilisés et qui prétendent n'être pas rustres $\left.{ }^{18} »\right)$. Dans l'Art de la

Lettere, éditées par Francesco Gaeta, Milan, Feltrinelli, 1981, p. 48-50 : "parmi les différentes choses qui démontrent ce qu'un homme vaut, il n'est pas à sous-estimer de voir comment il est facile à croire ce qu'on lui dit et prudent à simuler ce dont il veut persuader les autres; de telle manière que, chaque fois que quelqu'un croit ce dont il ne doit pas croire et simule mal ce dont il veut persuader, on peut le définir inconsistant et sans aucune prudence "; sur cette lettre, cf. Elena Fasano Guarini, Repubbliche e principi. Istituzioni e pratiche di potere nella Toscana granducale del '500-600, Bologne, Il Mulino, 2010, p. 128 et Sandro Landi, Le regard de Machiavel. Essai d'histoire intellectuelle, Rennes, Presses Universitaires de Rennes, 2020.

16 Machiavel, Euvres, cit., p. 216.

17 Ibid., p. 123 : « car, outre les choses énoncées ci-dessus, la nature des peuples est changeante : et il est facile de les persuader d'une chose, mais difficile de les maintenir dans cette persuasion. Aussi faut-il être organisés de façon telle que, lorsqu'ils ne croient plus, on puisse les faire croire de force".

18 Ibid., p. 215, sur ce chapitre des Discours, cf. Michele Lodone, «Savonarola e Machiavelli: una 
guerre, il est affirmé que la capacité oratoire du capitaine est le seul remède capable de détourner une multitude d'une idée ou d'une image (" opinion sinistre ") acceptée comme vraie mais contraire " au bien commun ou à votre opinion $^{19}$ ». Dans Discours (I, 14), il est précisé que le noyau du discours persuasif chez les Romains était religieux et relatif, en particulier, aux pratiques divinatoires ("jamais [les Romains] ne seraient partis pour une expédition sans avoir persuadé les soldats que les dieux leurs promettaient la victoire $\left.{ }^{20} »\right)$.

La question de la persuasion est étroitement liée à la maîtrise et à l'interprétation de signes. Qui interprète et comment ? À première vue, l'acte d'interpréter et, selon les nécessités, de manipuler les signes semble être l'une des prérogatives qui distingue le petit nombre du grand nombre, les " hommes sages » du commun. La lecture de Discours I, 14, consacré à la façon dont, pendant la guerre contre les Samnites, le consul Papirius dissimule des présages négatifs afin de maintenir intact le moral des troupes semble confirmer cette hypothèse ${ }^{21}$. De même, le passage bien connu de Discours I, 12 dans lequel il est dit que " les chefs d'une république ou d'un royaume " doivent " favoriser et développer toutes les mesures utiles à la religion, quand bien même ils en connaîtraient la fausseté ", a permis d'assimiler le comportement de cette élite à la figure du "noble mensonge " présent notamment au livre III de la République de Platon ${ }^{22}$.

Cette lecture est sans doute cohérente avec le texte machiavélien mais elle ne considère qu'un aspect de son analyse : en effet, l'interprétation est, selon Machiavel, une compétence que " les hommes sages " sont obligés de partager avec le peuple. Un collectif se démontre en effet particulièrement compétent dans la lecture des signes, et dans au moins un passage de l'Art de la guerre est mis en évidence le conflit d'interprétation qui s'établit entre la masse des soldats qui interprète "de façon sinistre " différents types de signes (foudres, éclipses, tremblements de terre, accidents inexplicables...) et ses chefs qui se retrouvent dans l'obligation d' " interpréter en leur faveur les augures sinistres ", en d'autres termes, de donner de ces mêmes signes une lecture divergente mais tout de même cohérente avec les croyances de la multitude ${ }^{23}$. Cette lecture

nota su Discorsi I, 11 », Interpres, vol. 30, 2011, p. 284-298.

19 Ibid., p. 555.

20 Ibid., p. 220.

21 Cf. John M. Najemy, "Papirius and the Chickens, or Machiavelli on the Necessity of Interpreting Religion ", Journal of the History of Ideas, $n^{\circ}$ 2, vol. 60, 1999, p. 659-681.

22 Platon, La République, traduction et présentation par Georges Leroux, Paris, Flammarion, p. 169 (III, 389); sur cette lecture, cf. Carlo Ginzburg, A distance. Neuf essais sur le point de vue en histoire, Paris, Gallimard, 2001, p. 55 ; la question du " noble mensonge » est nodale chez Leo Strauss, La persécution et l'art d'écrire, Paris - Tel Aviv, Éditions de l'Éclat, 2003, p. 40-41; sur Machiavel théoricien de la doctrine averrö̈ste de la "double vérité ", cf. Alberto Tenenti, "La religione di Machiavelli », Studi storici, n 1, vol. 10, 1969, p. 709-748.

23 Machiavel, Euvres, cit., p. 589 : « les capitaines antiques connaissaient une difficulté dont ceux d'aujourd'hui sont presque libérés : elle consistait à interpréter en leur faveur de mauvais 
d'en bas des présages est techniquement qualifiée par Machiavel de « superstitieuse ", un terme rare, qu'il n'emploie que deux fois, précisant que le christianisme (" notre religion ») combat ces pratiques ${ }^{24}$. C'est une observation qui n'a pas été remarquée mais qui est importante : Machiavel semble se rendre compte que le christianisme, agissant sur le substrat des croyances propres à la culture populaire, a restreint la possibilité de former des interprétations divergentes d'évènements extraordinaires et facilité, en dernière analyse, la démarche persuasive des "hommes sages». Nous reviendrons sur ce point, mais il est essentiel de souligner pour l'instant que, quoique variable dans le temps, Machiavel reconnaît au peuple une culture religieuse et des compétences dans la lecture des signes autonomes et non complètement manipulables.

\section{Le pacte de croyance}

Pour comprendre la spécificité de la position de Machiavel sur la religion en tant qu'expérience et patrimoine cognitif populaires, il est utile de procéder à une comparaison avec un texte contemporain qui décrit les pratiques religieuses d'une population amérindienne, les Tainos de l'île d'Hispaniola. La principale source à ce sujet sont les décades De orbe novo (De orbe novo decades) de Pierre Martyr Anghiera, un ecclésiastique humaniste actif à la cour d'Espagne, l'un des premiers à offrir un récit complet des explorations des terres nouvellement découvertes à l'Occident ${ }^{25}$. La première décade (Oceana decas) est imprimée à Séville en 1511 ; il y a eu ensuite de nombreuses traductions partielles et manuscrites : l'une d'entre elles, relative à la religion des Tainos, envoyée par Giovanni Strozzi, citoyen de Ferrare, à Alessandro Zorzi au début $\mathrm{du} \mathrm{XVI}^{\mathrm{e}}$ siècle, se trouve à la Bibliothèque Nationale de Florence ${ }^{26}$.

présages. Car, si la foudre tombait sur l'armée, s'il y avait une éclipse de soleil ou de lune, s'il se produisait un tremblement de terre, si le capitaine tombait en montant ou descendant de cheval, ces signes étaient interprétés négativement per les soldats...» (Art de la guerre, VI).

24 Ibid. : "nombreux sont ceux qui ont expliqué les causes des éclipses de lune ou de tremblement de terre. Cela ne peut arriver à notre époque, car les hommes ne sont plus aussi superstitieux et que notre religion est contraire à de telles idées ".

25 Pierre Martyr Anghiera, De orbo novo decades, vol. I, Oceana decas, édition de Brigitte Gauvin, Paris, Les belles lettres, 2003.

26 Fede et superstitio et costumi de l'insula Spagnola, mandatami da Ferrara per messer Zuane de' Strozzi, BNCF, B.R. 234, publié - sans identifier sa source originale - dans Italian Reports on America, cit., p. 189-194. Sur Alessandro Zorzi et le fonds florentin contenant ses écrits, voir Plinio Freire Gomes, "Alessandro Zorzi e l'invenzione dei tropici ", in Diogo Ramada Curto, Angelo Cattaneo, André Ferrand Almeida (éds), La cartografia europea tra rinascimento e primo illuminismo, Florence, Olschki, 2003, p. 109-132. 
Se basant sur le témoignage de Ramón Pané, moine arrivé à Hispaniola à la suite de Colomb ${ }^{27}$, Pierre Martyr donne une description détaillée de ce culte, le premier, parmi ceux récemment découverts, à avoir ses propres dogmes, préceptes et rites et donc à pouvoir être techniquement défini comme une "religion ${ }^{28}$. Ce texte nous intéresse en particulier pour certains éléments de similitude avec les chapitres des Discours consacrés à la religion des Romains, en particulier I, 11 et I, 12, dont le plus important concerne la double structure qui sous-tend le fonctionnement de cette religion. De manière similaire au cas décrit par Machiavel, Pierre Martyr identifie une minorité (sapientiores) dotée de connaissances et de techniques permettant la diffusion de légendes complexes et incroyables qui sont reçues comme vérités de foi par une multitude spectatrice (" turbae simplici mirabundae suadent ») ${ }^{29}$. En outre, de même que la religion romaine, celle découverte à Hispaniola est basée sur le verdict d'oracles (Zemes), dont la lecture est confiée à une caste d'augures (Boitios). La capacité présumée de ces prêtres à dialoguer avec ces simulacres, considérée par Pierre Martyr comme une supercherie manifeste, est un expédient qui leur permet de se faire passer efficacement pour des prophètes et des guérisseurs, renforçant, en dernière analyse, la subordination politique du peuple ${ }^{30}$.

Il est bien sûr impossible de démontrer, en l'état actuel de la recherche, un contact direct entre ces deux textes. Cependant, il est plausible que Machiavel ait pris connaissance d'une ouvre - ou de parties de cette ouvre - qui dans son titre fait explicitement référence à Tite-Live, qui, de plus, circule en l'état de manuscrit à la cour pontificale et que Léon X montre d'apprécier ${ }^{31}$. On ne peut exclure que, dans une démarche comparative, Machiavel ait trouvé

27 Pour une édition récente de cette relation, cf. Ramon Pané, Relation de l’histoire ancienne des Indiens, Paris, La Différence, 2003.

28 Pierre Martyr Anghiera, De orbo novo decades, cit., p. 199 : «In Hispaniola insula nostri priusquam indigenas quicquam aliud quam caeli numina colere aut religionem ullam execere didicissent, diu versati sunt ".

29 Ibid., p. 205 : « Haec at alia huiusmodi multa pacato serenoque vultu sapientiores e podiis suggestiolisque turbae simplici mirabundae suadent, recitanque pro sacro "; dans la version de Giovanni Strozzi : "Siché in questo modo i lor sapienti con gravità et riputantia in lor pergoli e lochi eminenti dànno a intendere a li ditti insulani simpliciotti, a persuaderli il credere queste tal cose per vere e sacre ", in Italian Reports on America, cit., p. 191.

30 Pierre Martyr Anghiera, De orbo novo decades, cit., p. 209 : « His illos imbuunt superstitionibus eorum augures quos Bioitios vocant. Sunt et iidem medici qui plebeculae rerum insciae mille astruunt fraudes. Credere cogunt plebem hi augures, quia sunt apud eam auctoritatis egregiae, quos Zemes ipsos alloquantur futuraque praedicent "; dans la version de Strozzi : " et li sui sacerdoti àuguri, i quali li chiaman boviite, che li acostumano di tal superstition: et così sono i medici che insegnono a mile donne vecchie, insensate, plebee di mile fraude. Item i ditti àuguri che dicon parlar con zemes et che tuto sanno ", Italian Reports on America, cit., p. 192.

31 Brigitte Gauvin, "Introduction », p. XX-XXI ; per une étude consacrée à ce milieu, cf. Josephine Jungic, "Prophecies of the Angelic Pastor in Sebastiano del Piombo's Portrait of Cardinal Bandinelli Sauli and Three Companions ", in Majorie Reeves (éd.), Prophetic Rome in the High Renaissance period, Oxford, Clarendon Press, 1992, p. 345-370. 
dans ce cas ethnographique singulier la confirmation d'un modèle religieux et politique opérant dans des contextes spatiaux et temporels très différents. Mais cette question, dans l'ensemble, est secondaire ; il parait en revanche plus important de souligner ce qui permet de distinguer les deux cas.

Pierre Martyr identifie une structure de croyance unidirectionnelle, fondée sur la fraude et la contrainte ("Credere cogunt plebem »); Machiavel, au contraire, met en lumière une réalité plus complexe, basée sur la volonté collective de croire. La question des oracles, affrontée dans Discours I, 12, est essentielle à cet égard, car pour être crus, il est indispensable que ces derniers parlent une langue que le peuple identifie comme étant totalement autre par rapport à la sienne et surtout à celle des prêtres qui en administrent le culte. La manipulation à des fins de pouvoir de cette parole surnaturelle est une opération risquée car le peuple se démontre capable de découvrir les cas où la parole des oracles n'est en réalité que de la ventriloloquie faisant ainsi soudainement disparaitre l'autosuggestion collective qui rend possible l'acte de croire :

"Lorsque [les oracles] commencèrent à parler conformément à la volonté des puissants et que la fraude fut découverte par le peuple, les hommes devinrent incrédules $[\ldots]^{32} »$.

Autrement dit, le peuple est dépositaire d'un imaginaire religieux qui lui est propre - constitué de lieux, d'images, d'histoires - une donnée qui est authentique et qui lui permet, semble-t-il, de distinguer le vrai du factice et de reconnaître la manipulation. De ce point de vue, la description du " miracle " de la statue de Junon de Véies, rapportée par Tite-Live (V, 22), déjà commenté par Lorenzo Valla ${ }^{33}$, est extrêmement significative. Dans le récit de l'historien de Rome, un groupe de " jeunes gens choisis dans l'armée entière, le corps lavé et purifié, vêtus de blanc ", entrent dans le temple de la déesse de la cité étrusque conquise et croient la voir réagir à leurs mots et gestes. La relecture de ce passage proposée par Machiavel, met en évidence deux objets d'analyse distincts : le premier, cohérent avec le texte d'origine, concerne l'extraordinaire capacité d'autosuggestion de ce groupe, qui voit et entend ce qu'il estime être conforme à ses propres expectatives (" certains crurent voir qu'elle faisait des signes et d'autres qu'elle acceptait [...] ils crurent entendre la réponse qu'ils

32 Machiavel, Euvres, cit., p. 216.

33 Lorenzo Valla, La donation de Constantin, Paris, Les Belles Lettres, 2004, p. 91-92 : "Quant à la statue de Junon, il dit qu'elle donna un signe d'approbation, sans répondre, et que plus tard on compléta la fable en disant qu'elle avait parlé. Au sujet du signe de la tête encore, il est évident qu'ils ont menti car ils ont interprété que le mouvement de la statue s'était fait spontanément, tandis qu'ils l'enlevaient, ou ils ont imaginé, sur le même ton de plaisanterie qu'ils prenaient pour l'interroger, que la déesse de pierre, ennemie et vaincue, avait donné un signe d'approbation ; Tite-Live dit que les soldats s'écrièrent qu'elle avait fait ce signe, non qu'elle avait fait ce signe "; Le texte de Valla (1440) est publié à Strasbourg en 1506. 
attendaient à leur question $\left.{ }^{34} »\right)$. Le second, totalement réinventé, porte sur l'attitude des chefs militaires et politiques qui, pour tirer pleinement avantage de la situation, décident de se limiter à accompagner et encourager un processus considéré comme spontané (" cette opinion si crédule fut soutenue et développée par Marcus Furius Camille et les autres chefs de la cité »).

À bien des égards, la description du comportement du peuple florentin qui assiste au sermons de Savonarole et que ce dernier parvient à " persuader qu'il s'entretenait avec Dieu "(Discours I, 11) ${ }^{35}$, rentre dans ce même cadre d'analyse : il s'agit en effet d'un phénomène d'autosuggestion non pas provoqué, comme dans le cas précédent, par la fausse perception d'un objet (" le nombre de ceux qui le croyaient, sans qu'ils aient rien vu d'extraordinaire qui les ait porté à croire était infini »), mais par la seule volonté de se fier à un comportement et à une parole dotés de charisme ("sa vie, sa doctrine et surtout le texte dont il se servit suffisaient pour ajouter foi à sa parole»). Un détail significatif : dans la Première Décennale, un texte en vers publié en 1506, Machiavel décrit l'expérience collective vécue par le peuple spectateur des sermons de Savonarole d'une manière analogue : «inspiré par la puissance divine, vous enveloppa de ses paroles ${ }^{36}$ ". Machiavel semble donc classer le Dominicain dans la catégorie de ceux qui exercent la persuasion à travers la voix : Savonarole est donc, techniquement, un «enchanteur ».

En général, tous ces cas montrent que la persuasion ne peut se produire que dans une relation de réciprocité et que l'acte de croire est toujours le résultat d'un choix, jamais d'une simple imposition. Plus précisément, l'analyse que Machiavel consacre à la persuasion, met en lumière un processus fondé sur deux phénomènes psychiques basés sur l'ouïe et sur la vue : l'enchantement et la fascination (incantatiolfascinatio). La psychologie d'Aristote et d'Avicenne, objet d'étude et d'enseignement à Florence au début du XVI ${ }^{\mathrm{e}}$ siècle, traite ces phénomènes. Plus précisément, on trouve des analogies entre l'analyse de Machiavel et un texte publié à Florence entre 1504 et 1507, l'Opus de intellectu et de causis mirabilium effectuum du médecin d'Imola Andrea Cattani ${ }^{37}$. Les informations à son sujet sont rares ${ }^{38}$. Avec certitude Cattani est médecin

34 Machiavel, Euvres, cit., p. 216.

35 Ibid., p. 215.

36 Ibid., p. 1016.

37 Andreae Cattanii Imolensis Opus de intellectu et de causis mirabilium effectuum, sans lieu ni date de publication, imprimé probablement par Bartolomeo de’ Libri à Florence, voir la notice bibliographique, in http://opac.bncf.firenze.sbn.it/opac.

38 Comme le souligne Paola Zambelli, L'ambigua natura della magia. Filosofi, streghe, riti nel Rinascimento, Milan, Il Saggiatore, 1991, p. 71, qui a été la première à inscrire l'Opus de Cattani dans la tradition avicennienne; pour une première information sur Cattani, voir Carlo Colombero, "Andrea Cattaneo », in Dizionario biografico degli italiani, vol. 22, 1979 : http://www.treccani. it/enciclopedia/andrea-cattaneo_(Dizionario-Biografico). Pour une controverse qui l'a probablement opposé en 1501 à Léonard de Vinci, voir Martin Kemp, Lezioni dell'occhio: Leonardo da 
à l'hôpital de Santa Maria Nuova et professeur de philosophie à l'Université de Florence (Studium) entre 1499 et $1506^{39}$. Ici, comme il l'indique dans la dédicace de son ouvrage, il a commenté pendant un an (publice interpretmur), la psychologie (De Anima) d'Aristote ${ }^{40}$. Détail non négligeable, Cattani dédie son œuvre à Piero Soderini, soulignant le soutien du gonfalonier de la République de Florence - " doctorum virorum fautor " et "divinarum rerum cultor " - à cette réinterprétation d'Aristote largement conforme à l'interprétation d'Avicenne (Liber de Anima) et à l'orthodoxie religieuse ${ }^{41}$. Une partie substantielle du traité de Cattani est consacrée à exposer des phénomènes de suggestion qui sont transmis par la parole (incantationes) ou par la vue (fascinationes). Dans les deux cas, Cattani tient compte, d'une part, de l'intention à opérer l'artifice et, d'autre part, de la volonté de l'accepter. En d'autres termes, il aborde la question de la persuasion et de la crédulité en termes médicaux e il pense que, dans la mise en ouvre de ce dispositif psychique, est indispensable le concours (" maximam habentis fiduciam») des deux parties (fascinans/fascinatus). Cattani traite essentiellement des cas individuels ; à une autre échelle d'observation, donc, non plus individuelle mais collective, Machiavel met en évidence le pacte tacite de croyance qui lie enchanteurs et enchantés, fascinateurs et fascinés, et que des facteurs externes, bien que minimes, peuvent, à tout moment, remettre en question.

\section{Instrumentum regni?}

"Grand' chose certes et toutesfois si commune qu'il s'en faut de tant plus douloir et moins s'esbahir, voir un million d'hommes servir miserablement aiant le col sous le joug non pas contrains par une plus grande force, mais aucunement (ce semble) enchantés et charmés par le nom seul d'un, duquel ils ne doivent ni craindre la puissance puis qu'il est seul, ny aimer les qualités puis qu'il est en leur endroit inhumain et sauvage ${ }^{42}$ ».

Vinci discepolo dell'esperienza, Milan, Vita e Pensiero, 2004, p. 192 et The literary works of Leonardo da Vinci, II, édité par Carlo Pedretti, Londres-New York, Phaidon press, 1977, p. 132. Pour un parallèle entre Cattani et Pomponazzi, cf. Francesco Piro, "Sull'antropologia dei rudes prima di Vico. Immaginazione, credulità, passionalità ", Laboratorio dell'ISPF, vol. 1, 2005, p. 337-369.

39 Cfr. Armando Verde, Lo Studio fiorentino, 1473-1503, II, Ricerche e documenti, Florence, Istituto Nazionale di studi sul Rinascimento, 1973, p. 20-21 et Nancy G. Siraisi, Medicine \& the Italian Universities, 1250-1600, Leiden, Boston, Köln, Brill, 2001, p. 236.

40 Andrea Cattani, Opus de intellectu, cit., p. 3.

41 Sur ce texte dans le cadre du débat sur Avicenneà Florence, voir Eugenio Garin, Dal Rinascimento all'Illuminismo. Studi e ricerche, Pise, Nistri-Lischi, 1970, p. 60-61 et Nancy G. Siraisi, Medicine o the Italian Universities, cit., p. 235.

42 Étienne De La Boétie, Discours de la servitude volontaire ou Contr'un, Paris, Gallimard, 1993, p. 79-80. 
Lecteur de Machiavel ${ }^{43}$, Étienne de La Boétie comprend que la persuasion - à son tour fondée sur la capacité à " charmer " un nombre potentiellement infini d'individus - est la clé de l'assujettissement volontaire ${ }^{44}$. Il convient de noter qu'avec le temps, cette clé de lecture de Machiavel est devenue inopérante. Celle qui domine aujourd'hui voit plutôt chez Machiavel un exemple classique d'utilisation frauduleuse, à des fins d'assujettissement, de la religion ${ }^{45}$. Toutefois, si nous prêtons attention à la complexité de l'analyse machiavélienne de la persuasion, la formule instrumentum regni, souvent employée pour définir la position de Machiavel à l'égard du phénomène religieux, nous apparaît comme réductrice et anachronique. Elle est réductrice car elle ne prend en compte qu'un seul aspect du processus, c'est-à-dire la volonté de domination des " hommes sages "; elle est anachronique, car plus utile, sans doute, à saisir la manière dont, au XVII ${ }^{\mathrm{e}}$ siècle, les théoriciens du libertinisme ont lu Machiavel qu’à comprendre comment ce dernier a conçu le phénomène religieux. Et rien n'est en effet plus efficace pour se rendre compte de la distance qui sépare Machiavel de ses lecteurs libertins qu'une confrontation sur la question de la persuasion. Gabriel Naudé entre autres, traite de ce thème au chapitre IV des Considérations politiques sur les coups d'État publiées pour la première fois à Rome en $1639^{46}$. La mise en ouvre du coup d'État est liée, selon Naudé, à la persuasion et, plus précisément, à la capacité d'une minorité sans scrupules d'activer la crédulité de la multitude en mobilisant

43 Cf. Jean Balsamo, "Le plus meschant d'entre eux ne voudroit pas estre Roy” La Boétie et Machiavel ", Montaigne Studies, vol. 11, 1999, p. 5-27.

44 Voir à ce sujet les observations de Thierry Ménissier, «Lefort lecteur de Machiavel : le travail continué de l'œuvre », "Revue Française d'Histoire des Idées Politiques », n² 2, vol. 46, 2017, p. 9-32, à la p. 14.

45 Pour une synthèse de cette interprétation cf. Marco Geuna, "Ruolo dei conflitti e ruolo della religione nella Riflessione di Machiavelli sulla storia di Roma ", in Roberto Caporali, Vittorio Morfino, Stefano Visentin (éds), Machiavelli: tempo e conflitto, Milan-Udine, Mimesis, 2013, p. 107-139.

46 Gabriel Naudé, Considérations politiques sur les coups d'État, Paris, Éditions de Paris, 1988, p. 147-172: «De quelles opinions faut-il être persuadé pour entreprendre les coups d'état » et Jean-Pierre Cavaillé, "Gabriel Naudé : destinations et usages du texte politique ", Cahiers $d u$ Centre de Recherches Historiques, vol. 20, 1998, p. 1-8. 
des images et des récits à caractère religieux ${ }^{47}$. La passivité extrême du peuple $e^{48}$ et le caractère unidirectionnel de ce processus témoignent de l'écart qui s'est produit, en un peu plus d'un siècle, entre Naudé et sa source machiavélienne ; la généalogie qu'une partie de la critique contemporaine tend à établir entre Machiavel et le mouvement libertin paraît donc faiblement fondée sur des raisons d'ordre philologique ${ }^{49}$.

Pour aboutir à un point de vue non anachronique sur ce problème, il serait en revanche nécessaire de le contextualiser à la lumière de débats contemporains ${ }^{50}$. Par exemple, la surprenante proximité des positions exprimées par Machiavel dans les Discours et Pietro Pomponazzi dans le De naturalium effectuum causis sive de incantationibus (1520) au sujet de l'origine naturelle des miracles ${ }^{51}$ renvoie à un contexte commun : la tradition médicale péripatéti-

47 Comme le montre en particulier ce passage (ibid., p. 159-160) qui est un véritable patchwork machiavélien : «Finalement pour ce qui est de la politique il faut [...] montrer en quelle façon les princes et leurs ministres, qui font leur profit des esprits adonnés à la bigoterie (Tite-Live) ont bien su ménager la religion, et s'en servir comme du plus facile et plus assuré moyen qu'ils eussent pour venir au bout de leurs entreprises plus relevées. Je trouve donc qu'ils en ont usé de cinq façons principales, sous lesquelles on en peut rapporter beaucoup d'autres petites. La première et la plus commune et ordinaire est celle de tous les législateurs et politiques qui ont persuadé à leurs peuples d'avoir la communication des dieux, pour venir plus facilement à bout de ce qu'ils avaient la volonté d'exécuter : comme nous voyons qu'outre ces Anciens que nous avons rapportés ci-dessus, Scipion voulut faire croire qu'il n'entreprenait rien sans le conseil de Jupiter Capitolin, Sylla que toutes ses actions étaient favorisées pas Apollon de Delphes duquel il portait toujours une petite image ; et Sertorius que sa biche lui apportait du les nouvelles de tout ce qui était conclu dans les concile des dieux. Mais pour venir aux histoires qui nous sont plus voisines, il est certain que par des semblables moyens [...] Jérôme Savonarole [domina] à Florence, duquel nous avons cette remarque dans Machiavel (sur Tite-Live) : Le peuple de Florence n'est pas bête, auquel néanmoins frère Jérôme Savonarole a bien fait croire qu'il parlait avec Dieu ».

48 Ibid., p. 156 : «Que Postel lui [au peuple] persuade que Jésus-Christ n’a sauvé que les hommes et que sa mère Jeanne doit sauver les femmes, il le croira soudain. Que David George se dise fils de Dieu, il l'adorera. Qu'un tailleur enthousiaste et fanatique contrefasse le roi dans Münster et dise que Dieu l'a destiné pour châtier toutes les puissances de la terre, il lui obéira et le respectera comme le plus grand monarque du monde ".

49 Cf. Jean-Pierre Cavaillé, "Libérer le libertinage. Une catégorie à l'épreuve des sources ", Annales HSS, 64 (2009), p. 45-78; les lectures néo-libertines de Machiavel rentrent à plein titre dans le problème analysé par Pier Paolo Portinaro, Le mani su Machiavelli. Una critica dell'Italian theory, Rome, Donzelli, 2018.

50 Pour une première reconstitution de ce débat sur la tradition avicennienne entre la fin du $\mathrm{XV}^{e}$ et le début du XVI ${ }^{e}$ siècle, voir Piro Francesco, "Sull'antropologia dei «rudes» ", cit., p. 349-351.

51 Machiavel, Euvres, cit., p. 216 (Discours, I, 12) et Pietro Pomponazzi, De incantationibus, édité par Vittoria Perrone Compagni, Florence, Olschki, 2011 (caput decimum, p. 67-113). Pomponazzi, professeur de philosophie à Bologne depuis 1511, publie le De incantationibus sous forme manuscrite en 1520, cf. Vittoria Perrone Compagni, "Pietro Pomponazzi ", in Dizionario biografico degli italiani, vol. 84, 2015 : http://www.treccani.it/enciclopedia/pietropomponazzi_(Dizionario-Biografico). Pour une étude du De incantationibus à la lumière de la tradition médicale aristotélicienne, voir Tristan Dagron, "La doctrine des qualités occultes dans le De incantationibus de Pomponazzi ", Revue de métaphysique et de morale, n 1, vol. 49, 2006, p. 3-20. L'étude des rapports entre Machiavel et Pomponazzi, non seulement en termes 
cienne, largement confiée à l'enseignement dans les universités de Padoue et de Bologne et à la circulation manuscrite. Mais la manière dont un débat a pu réellement se constituer autour de ces thèmes nous échappe encore largement. Pourtant, c'est à la lumière de ce débat qu'il deviendrait pertinent de se demander en quoi l'analyse machiavélienne de la religion est originale. On peut toutefois tenter une hypothèse : Cattani et Pomponazzi appliquent à la religion des clés d'interprétation typiques de la psychologie aristotélicienne et avicennienne ; à partir de cette base, Machiavel identifie dans la religion un champ de phénomènes qui permet d'étudier, de façon privilégiée, la nature mentale de la subordination politique.

\section{«Il est mal de dire du mal du mal »}

Dans cette perspective, il est enfin possible de revoir le lieu commun de la critique selon lequel le christianisme, religion fondée sur des valeurs faibles, aurait contribué à affaiblir le lien politique. Bien entendu, si le terme de comparaison entre le monde ancien et le monde moderne est constitué - comme nous le lisons dans Discours II, 2 - par la propension des "peuples » à la liberté, l'avènement et la diffusion du christianisme correspondent en effet à une mutation qui a rendu la liberté, en tant que pratique politique et patrimoine de valeurs, très probablement révolue ${ }^{52}$. Mais si la question est aussi et avant tout de comprendre la capacité des religions à construire le "sentiment d'obligation politique ${ }^{53}$ ", l'analyse menée dans les Discours met indéniablement en évidence une fonctionnalité spécifique du christianisme.

Machiavel illustre dans ce chapitre la double transformation anthropologique déterminée par la religion du Christ : la première est liée à «l'éducation ", c'est-à-dire au système de valeurs qu'elle a introduit ${ }^{54}$; la seconde concerne

d'histoire des idées mais aussi de contacts possibles, directs ou indirects, reste presque entièrement à faire, pour quelques indications en ce sens, cf. Paul A. Rahe, Against Throne and Altar: Machiavelli and Political Theory Under the English Republic, Cambridge, Cambridge University Press, 2008, p. 97 et Alison Brown, The Return of Lucretius to Renaissance Florence, Cambridge (Mass.) et Londres, Harvard University Press, 2010, p. 76 ; pour l'histoire de ce rapport à la lumière de la tradition libertine, voir Vittorio Frajese, " Note su Machiavelli, editoria e cultura nell'Italia del Rinascimento e della Controriforma ", Studi storici, n 2, vol. 38, 1997, p. $135-155$.

52 Machiavel, Euvres, cit., p. 298 (Discours, II, 2) « Me demandant donc d'où il peut provenir qu'en ces temps anciens les peuples étaient plus attachés à la liberté qu'aujourd'hui, je crois que cela provient de la même cause qui rend aujourd'hui les hommes moins courageux. Je crois que c'est la différence existant entre notre éducation et celle des Anciens, qui provient de la différence entre notre religion et celle des Anciens » et les observations sur ce point de Emanuele Cutinelli-Rèndina, "Religione ", cit., p. 392-400.

53 Emanuele Cutinelli-Rèndina, Chiesa e religione in Machiavelli, Pise-Rome, Istituti editoriali e poligrafici internazionali, 1998, p. 222.

54 Sur la notion d'" éducation " dans ce chapitre, cf. John M. Najemy, "Papirius and the Chickens ", cit., p. 667. 
plutôt la perception du sacré. En substance, si dans les religions anciennes l'adhésion aux cultes se faisait dans un contexte de spectacularisation du sacrifice " sanglant et horrible » qui, par mimétisme, touchait les sens et " rendait les hommes pareils à lui ", le christianisme, religion plus " délicate », recherche et exige un consentement intime dont la nature est essentiellement mentale ${ }^{55}$.

La révolution perceptive produite par le christianisme permet de penser à de nouvelles techniques de gouvernement de l'imaginaire individuel et collectif. Dans son analyse du système de valeurs et de croyances ("façon de vivre ») introduit par la nouvelle religion, Machiavel s'attarde (principalement dans Discours, II, 2 et III, 1) ${ }^{56}$, sur la foi dans le paradis, le culte des saints, la confession et la prédication : un arsenal de pratiques et d'objets de foi, sans égal dans le monde ancien, qui a permis au christianisme non seulement de triompher du paganisme mais aussi de survivre à sa crise au XIII ${ }^{e}$ siècle et de se réformer. La question classique de Leo Strauss, " comment Machiavel pouvait-il sur la base de ses propres principes, s'accommoder de la victoire du christianisme » trouve probablement ici une réponse convaincante ${ }^{57}$.

Certes, Machiavel ne manque pas de souligner l'utilisation frauduleuse de ces objets et pratiques par une caste de religieux qui reste étrangère aux croyances du peuple ( «ils fontainsi tout le mal qu'ils peuvent parce qu' ils ne craignent pas des punitions qu'ils ne voient ni ne croient »). Mais, après tout, c'est une attitude qui n'est pas spécifique au christianisme et qui peut permettre une comparaison entre les ministres du culte chrétien et ceux d'autres religions, éloignés dans le temps ou dans l'espace. L'essentiel est donc ailleurs : surtout dans l'extraordinaire capacité de persuasion

55 Machiavel, Euvres, cit., p. 299.

56 Machiavel, Euvres, cit., p. 299 et 372 : «Et si notre religion requiert que l'on soit fort, elle demande aussi que l'on soit plus apte à supporter la souffrance qu'à accomplir des choses fortes. Cette manière de vivre semble donc avoir affaibli le monde et l'avoir livré aux scélérats qui peuvent le gouverner en toute tranquillité, car ils voient que l'ensemble des hommes, pour aller au Paradis, pense davantage à supporter leurs coups qu’à s'en venger "; "quant aux religions, on voit aussi que ces rénovations sont nécessaires, à l'exemple de la nôtre. Si elle n'avait pas été ramenée à ses origines par saint François et saint Dominique, elle se serait totalement éteinte. Car ceux-ci, par la pratique de la pauvreté et l'exemple de la vie du Christ, la ramenèrent dans l'esprit des hommes [nella mente degli uomini], où elle s'était éteinte. Leurs nouvelles institutions furent si fortes que la licence des prélats et des chefs de l'Église ne la feraient pas s'effondrer. Vivant encore pauvrement ces ordres ont tant de crédit auprès du peuple grâce aux confessions et aux prédications qu'il lui font croire qu'il est mal dire mal du mal, qu'il est bien de vivre sous leur autorité et, si les hommes commettent des fautes, de laisser Dieu les châtier. Ils font ainsi tout le mal qu'ils peuvent, parce qu'ils ne craignent pas les punitions qu'ils ne voient ni ne croient. Un tel renouveau a donc maintenu et maintien cette religion ".

57 Leo Strauss, Pensées sur Machiavel, Paris, Payot, 2007, p. 308 ; Carlo Ginzbug, Nondimanco. Machiavelli, Pascal, Milan, Adelphi, 2018, p. 155 : «l'importanza della domanda formulata da Strauss [...] è fuori discussione; ma trovare una risposta è forse impossibile " ("l'importance de la question formulée par Strauss [...] est incontestable ; mais trouver une réponse est peut-être impossible»). Pour une étude de ce processus cohérente avec les analyses de Machiavel, cf. Peter Brown, Pouvoir et persuasion dans l'Antiquité tardive, vers un empire chrétien, Paris, Le Seuil, 1998. 
offerte par le christianisme, une religion qui opère un déplacement de l'imaginaire collectif vers des lieux (paradis/enfer), des icônes (saint François, saint Dominique), vers un système des récompenses et de sanctions immatérielles, capables de s'enraciner par la seule force de la suggestion, écrit-il, probablement avec une acception technique, "dans l'esprit des hommes" (nella mente degli uomini). Il s'agit donc d'un processus d'adhésion rendu possible par la volonté de faire croire, alimenté, comme l'observe frère Timothée dans la Mandragore, par un culte incessant des images $^{58}$, ainsi que, rappelons-le, par la lutte contre les "superstitions ", qui rend marginales les interprétations populaires de ces mêmes objets de foi ; mais ce processus serait inopérant sans que la majorité des individus manifeste, par intime conviction, une volonté de croire (" pour aller au paradis, l'ensemble des hommes pense davantage à supporter leurs coups qu'à s'en venger ").

Sans aucun doute, Machiavel saisit l'extraordinaire potentialité politique de cette religion qui, sans exemples comparables dans le monde ancien et moderne, permet non seulement d'inhiber les conflits politiques et sociaux, mais aussi d'inaugurer des formes d'autocensure extrêmement pénétrantes, capables d'intervenir sur les racines mentales du dissentiment (" ils leur font croire qu'il est mal de dire du mal du mal»). Avec certitude, cet aspect n'a pas échappé à l'attention d'un lecteur attentif de ces pages, Giovanni Botero, qui, quelques décennies plus tard, décrit ainsi le gouvernement des consciences que seule la religion du Christ permet de réaliser avec une efficacité redoutable :

«Parmi toutes les lois, la plus favorable au prince est la loi chrétienne ; car elle permet non seulement de soumettre les corps et les biens des sujets mais également les esprits et les consciences et elle lie non seulement les mains mais aussi les sentiments et les pensées $"^{59}$.

\author{
Sandro Landi \\ EA $4574 \mathrm{SPH}$ \\ Université Bordeaux Montaigne \\ Sandro.Landi@u-bordeaux-montaigne.fr
}

58 Machiavel, Euvres, cit., p. 1139 : "J'ai dit les matines, j'ai lu une Vie des saints Pères, je suis allé à l'église, j’ai allumé une lampe qui était éteinte, j’ai changé le voile d'une sainte Vierge qui fait des miracles. Combien de fois ai-je répété aux moines de la tenir propre ! Et puis ils s'étonnent si la dévotion baisse! Je me souviens du temps où il y avait cinq-cents ex-voto, et aujourd'hui il n'y en a qu'une vingtaine. C'est notre faute aussi, nous n'avons pas su maintenir leur réputation " ( $\mathrm{La}$ Mandragore, acte cinquième, scène première) ; pour une lecture anthropologique de ce passage, cf. Jane Garnett et Gervase Rosser (éds), Spectacular Miracles: Transforming Images in Italy from the Renaissance to the Present, Londres, Reaktion Books, 2013, p. 109-110: "Machiavelli captured here, in part, the vital element of a different kind of dramatic ritual in the activation of an image cult ».

59 Giovanni Botero, Della Ragion di Stato, Bologne, Cappelli, 1930 [1589], p. 94 ; sur le « gouvernement des consciences ", cf. Adriano Prosperi, Tribunali della coscienza. Inquisitori, confessori, missionari, Turin, Einaudi, 1996. 
Historien spécialiste de l'Italie moderne, Sandro Landi est professeur à l'Université Bordeaux Montaigne, directeur de l'École doctorale "Montaigne Humanités" et de la revue Essais. La traduction de son dernier ouvrage sur Machiavel (Le regard de Machiavel. Essai d'histoire intellectuelle), va paraître en 2020 aux Presses Universitaires de Rennes.

\title{
Résumé
}

Il y a environ cinquante ans, un grand spécialiste d'histoire religieuse, Delio Cantimori, affirmait que dans les écrits de Machiavel, " le thème religieux " est celui qui est " le moins explicitement défini et le moins clairement expliqué ". Malgré cela, la question de la religion chez Machiavel est devenue progressivement un thème classique d'étude et de débat. Dans cet article, je me propose de l'aborder sous un angle qui n'a pas été suffisamment exploré même s'il constitue l'un des plus constants et récurrents objets d'analyse et d'écriture de Machiavel : celui de la religion comme expérience ou, plus précisément, comme noyau d'expériences partagées, dans un temps et dans un espace historique déterminés, par une communauté ou un par un collectif. Le but est celui de contribuer à la définition d'une phénoménologie de la croyance chez Machiavel.

\section{Mots-clés}

Machiavel, religion, croyance, persuasion.

\begin{abstract}
About fifty years ago, a great scholar of religious history, Delio Cantimori, stated that in Machiavelli's writings, "the religious theme" is the one that is "least explicitly defined and least clearly explained". Despite this, the question of religion in Machiavelli's writings has gradually become a classic theme of study and debate. In this article, I propose to approach it from an angle that has not been suffciently explored even though it constitutes one of the most constant and recurrent objects of analysis and writing by Machiavelli: that of religion as experience or, more precisely, as a nucleus of shared experiences, in a given historical time and space, by a community or a collective. The aim is to contribute to the definition of a phenomenology of belief in Machiavelli.
\end{abstract}

\section{Keywords}

Machiavelli, religion, belief, persuasion. 